\title{
An Age-Period-Cohort And Joinpoint Analysis Of Colorectal Cancer Incidence Trends in Ho Chi Minh City, Vietnam(1996-2015)
}

\section{Dung X. Pham}

Ho Chi Minh City Oncology Hospital

An HT. Phung

Pham Ngoc Thach University of Medicine

Tung D. Bui

Ho Chi Minh City Oncology Hospital

Linh D. Mai

Pham Ngoc Thach University of Medicine

Thach S. Tran

Garvan Institute of Medical Research

Lan T. Ho-Pham

Pham Ngoc Thach University of Medicine

\section{Tuan V. Nguyen}

Ton Duc Thang University

Hien D. Nguyen ( $\nabla$ ndhien.stu13@medvnu.edu.vn )

Pham Ngoc Thach University of Medicine

\section{Research Article}

Keywords: Cancer, CRC , APC

Posted Date: April 14th, 2021

DOI: https://doi.org/10.21203/rs.3.rs-396679/v1

License: (c) (1) This work is licensed under a Creative Commons Attribution 4.0 International License. Read Full License 


\section{Abstract}

\section{Background}

Little is known about the recent trends in colorectal cancer (CRC) in Vietnam.

\section{Methods}

Based on the Ho Chi Minh City Cancer Registry data during 1996-2015, we calculated the annual rate of change (ARC) using Joinpoint regression analysis and performed the age-period-cohort (APC) analysis using the United States National Cancer Institute's web-based statistical tool.

\section{Results}

During 1996-2015, the overall age-standardized incidence rate of CRC increased from 10.5 to 17.9 per $100,000$. CRC incidence elevated more rapidly in men (ARC $=4.7,95 \% \mathrm{Cl}: 2.2-7.3)$ than women (ARC = 2.6, 95\% Cl: 0.6-4.8). The highest and lowest increasing ASR observed in the 50-64 years old group (ARC $=5.3,95 \% \mathrm{Cl}: 2.8-7.9)$ and $<50$ years old group ( $\mathrm{ARC}=1.1,95 \% \mathrm{Cl}:-0.7-2.9)$, respectively. Regarding subsites, rectal cancer had the highest rate increase (ARC $=3.3,95 \% \mathrm{Cl}: 1.0-5.7)$. Furthermore, the APC analysis indicated significant increases in CRC incidence of recent birth cohorts from 1976-1980 in either gender.

\section{Conclusions}

The CRC incidence in Ho Chi Minh City increased with the more prominent rising rates among men, older people, and rectal subsites. This study's result implied the need of launching a population-based CRC screening program in Ho Chi Minh City.

\section{Background}

Globally, colorectal cancer (CRC) is the third most frequently diagnosed cancer and the second most common oncological cause of death in both genders, with an estimated 1,849 million new cases of CRC and 880,792 deaths attributable to CRC in $2018^{1}$.

Asian countries have been regarded as having a lower risk for CRC compared to Western countries ${ }^{2}$. However, there have been continuously sharp increases in CRC incidence in the Asian population during the past decades, including Japan, South Korea, China, Singapore, Hong Kong, and Taiwan, with a two to four-fold increase ${ }^{3-5}$. This situation is recognized as a marker of the 'cancer transition' in which developing countries undergo high-rate cancers typical in high-income nations. These high rates particularly link to the rising prevalence of westernized lifestyles, such as unhealthy diet, obesity, physical inactivity, and smoking ${ }^{6,7}$. 
Otherwise, CRC has been deemed a disease burden only among older individuals as CRC development rates elevate significantly after age 50 years $^{8}$. Notwithstanding, several recent studies indicated notably increasing incidences of early-onset colorectal cancer in high-income countries, such as the USA, the UK, Australia, Canada, Denmark, New Zealand, and Norway ${ }^{9-13}$. Early-onset CRC may have a family history or genetic predisposition; however, the majority of cases observed (approximately $75 \%$ ) are sporadic ${ }^{7}$. As many Asian nations being in line with the trend of CRC in Western populations, it could be expected a similar rising of early-onset CRC in Asia.

In Vietnam - one of the developing countries in Asia, we lack analysis of the recent secular trends in colorectal cancer incidence by age, sex, and tumor subsite on either a regional or national scale. It is still unclear whether colorectal cancer in Vietnam has a similar steep upward trend with many other Asian Countries surveyed and be affected by the rapid cancer shift. Therefore, this study aimed to investigate the trends in epidemiology and anatomical subsites of CRC in Ho Chi Minh City, Vietnam, from 1996 to 2015. Additionally, we performed an age-period-cohort analysis to estimate the contributions of age, period, and cohort effects on the observed trend.

\section{Methods}

\section{Data source}

With the authorization of the Ethics Committee of the Oncology Hospital of Ho Chi Minh City, this population-based study was conducted extracting data from the Ho Chi Minh City Cancer Registry from 1996 to 2015 and performed in accordance with the relevant guidelines and regulations. All datasets were anonymized before analysis and the need of the written informed consent has waived by the Ethics Committee of the Oncology Hospital of Ho Chi Minh City.

Registration data were collected and maintained according to guidelines from the International Agency for Research on Cancer and the International Association of Cancer Registries for low and middle-income countries $^{14}$. The Ho Chi Minh City Cancer Registry used the ICD-10 code for colon (C18.0-C18.9 and $\mathrm{C} 26.0$ ) and rectum (C19.9 and C20.9) diagnosis. The colon cancer anatomical subsites were further classified following the International Classification of Diseases for Oncology, third edition (ICD-0-3) as proximal (C18.0 and C18.2-C18.5), distal (C18.6 and C18.7), or others (C18.1, C18.8, C18.9, and C26.0) ${ }^{15}$. The others subsite were excluded from the subsite-specific analyses.

Population census between 1996 and 2015 was retrieved from the General Statistics Office (GSO) of Ho Chi Minh City and the Bureau of Statistics of Vietnam.

\section{Statistical analysis}

From the population statistics of Ho Chi Minh City and the Bureau of Statistics of Vietnam, we examined CRC incidences for four consecutive periods (1996-2000, 2001-2005, 2006-2010, and 2011-2015). 
Owing to some low case counts, we aggregated the data every five years, which would enhance the stability of statistics.

The age-standardized incidence rates (ASRs) per 100,000 population were calculated by direct standardization, using the Segi's world population. The CRC rate trends were stratified by age groups $(<50,50-64,65-79$, and $80+)$, sex, and anatomical subsites.

We used the Joinpoint regression software version 4.8 to performed the Joinpoint regression analysis ${ }^{16}$. Joinpoint tests of significance use a Monte Carlo permutation method to determine the statistically significant changes across successive calendar periods, and the annual rate of changes (ARC) was estimated utilizing generalized linear models assuming a Poisson distribution ${ }^{17}$. Data were reported as mean (standard deviation, SD) for continuous variables, and frequency and percentage for categorical variables.

For the age-period-cohort (APC) analysis, we used the United States National Cancer Institute's web-based statistical tool to evaluate the effects of age, period, and cohort on the CRC incidence ${ }^{18}$. Input data were the case and population counts for 12 five-year age groups $(30-34, \ldots, 80-84$, and $85+)$, and 4 five-year periods (1996-2000, 2001-2005, 2006-2010, and 2011-2015), spanning 15 partially overlapping fiveyear birth cohorts. The default age, period, and cohort for reference were the median of each category. The APC analysis parameters and functions included: longitudinal age curve, period and cohort rate ratios (RR), and local drifts. The longitudinal age curve depicts the fitted longitudinal age-specific rates in reference cohort adjusted for period deviations. Period RR are the ratios of age-specific rates in each period relative to the reference period, while cohort RR are the ratios of age-specific rates in each cohort relative to the reference cohort. Net drift is the analogue of the estimated ARC in the ASR. Local drift represents the estimated ARC over time specific to age group. Wald Chi-Square tests were used for hypothesis testing with two-sided $p$ values less than 0.05 were considered statistically significant.

\section{Results}

In total, 12,938 people were diagnosed with CRC in Ho Chi Minh City from 1996 to 2015 (Table 1), of whom 6,989 were men (53.9\%) and 5,970 were women (46.1\%). The age at diagnosis remained stable during the whole time, with the overall mean age of $60.0 \pm 15.0$. The patients' age were mainly $>50$ years

old $(n=9,882,76.4 \%)$. Both genders share a similar pattern in the shifting of age group distribution. In the first two calendar periods, the age group 65-79 had the largest proportion, but in the latter two calendar periods, the age group 50-64 emerged to be the most prevalent. Regarding topography, rectum was the most common subsite $(n=4,712,36.4 \%)$, followed by the others $(n=3,462,26.8 \%)$, proximal $(n=2,461$, $19.0 \%)$, and distal $(n=2,303,17.8 \%)$.

The overall ASR of CRC increased from 10.5 per 100,000 during 1996-2000 to 17.9 per 100,000 during 2011-2015, a 1.7-fold increase. This rise translates into an ARC of 3.8 (95\% confidence interval [95\% Cl]: 1.7 - 5.8). In men, the ASR elevated consistently from 12.6 to 24.6 per 100,000 between 1996-2015, 
corresponding to an ARC of 4.7 (95\% Cl: 2.2 - 7.3) (Table 2). In women, the ASR indicated a mild decrease from 9.2 to 7.8 per 100,000 between 1996-2005 before increased to 13.4 per 100,000 by the end of the study period (average ARC $=2.6,95 \% \mathrm{Cl}: 0.6-4.8$ ).

Fig. 1 displays the Joinpoint regression analysis of CRC incidence trends by age groups. Over the whole study period, all age groups had the elevating trend in the ASRs with the highest and lowest rates belonged to the $50-64$ years old group (average ARC $=5.3,95 \% \mathrm{Cl}: 2.8-7.9$ ) and $<50$ years old group (average $A R C=1.1,95 \% \mathrm{Cl}:-0.7-2.9)$, respectively. Only the $>80$ years old patients had the ASR increase continuously over time (ARC 3.6, 95\% Cl: $1.5-5.8$ ). Whereas, there was one detected joinpoint in the trend of each other three age groups: in 2005, 2006 and 2008 for $<50,50-64$ and 65-79 years old, respectively. Prior to the joinpoints, ASRs of CRC among patients in 50-64 and 65-79-years old age groups were stable with little decreasing or unchanged patterns. After that, the CRC rates rose steeply in both 5064 and 65-79 years-old cases. By the end of the period, the 65-79 age group took the lead of ASR among all patients (average ARC $=3.5,95 \% \mathrm{Cl}: 1.2-5.8$ ).

The Joinpoint regression analysis of the CRC incidence trends by anatomical subsites of the overall, men, and women is showed in Fig. 2a, Fig. 2b, Fig. 2c, respectively. Overall, all subsites observed an upward trend in ASRs. Rectal cancer had the highest rate increase with an average ARC of 3.3 (95\% Cl: $1.0-5.7)$, followed by the distal (ARC $=3.2,95 \% \mathrm{Cl}: 1.4-4.9)$ and proximal $(\mathrm{ARC}=1.5,95 \% \mathrm{Cl}: 0.1-3.0)$ colon cancer. Both genders shared the similar changing pattern of each subsites' rate with the overall.

The APC analysis parameters of CRC cancer in men and women are presented in Supplementary table 1. The longitudinal age curve, period and cohort RRs, and local drifts of CRC cancer in men and women are demonstrated in Fig. 3. The longitudinal age curves (Fig. 3a1 and Fig. 3a2) indicate that the CRC rates increased along with aging in both genders in the same birth cohort. The CRC rate was much higher in men than women from the age of 60-64 years old (Supplementary table 1). Local drift values were above 0 in most age groups of both genders, except for women aged 40-44 (Fig. 3b1 and Fig. 3b2). Both men and women reached the peak of local drift at ages of 55-59 years old. The period RRs had a similar pattern for both sexes: decreasing before the period of 2001-2005 and then sharply increasing with the same rate (Fig. 3c1 and Fig. 3c2). The cohort RRs indicated similar monotonic increasing trends over time in both genders with the similar rate (Fig. 3d1 and Fig. 3d2). Especially, men and women born from the 1976-1980 period increased the CRC risk strikingly. The Wald Chi-Square test indicated statistically significant for cohort and period effects, and the local drifts and net drift in both sexes ( $p<0.05$ for all).

\section{Discussion}

We found an upward trend of the CRC incidence with a higher rate increasing in men than women in Ho Chi Minh City, consistent with the patterns in other Asian countries over recent decades. However, our result differed from these countries - an appreciably higher rising occurred in the older than younger individuals. Regarding topography, the rectum accounted for the most prevalent anatomical subsite and had the highest rising rate in both genders. Furthermore, the APC analysis indicated significant period and 
cohort effects among both men and women, in which people born from the 1976-1980 period increased the $\mathrm{CRC}$ risk strikingly.

Many decades ago, CRC was rare in developing and low-risk areas such as Asia and was most common in high Human Development Index countries. In recent years, many studies reported the rapid increase of CRC incidence in Asia nations ${ }^{3-5}$. Till 2015, the ASR of CRC in South Korea, Singapore, Japan, Taiwan, and Hong Kong was 45, 33.7, 32.2, 45.3, and 38.4, respectively.

For the underlying reasons of the elevating trend in Ho Chi Minh City, the strong period and cohort effects among either gender in the recent timeframe suggest the increase in the prevalence of exposure to risk factors and improvements in diagnostic technique and healthcare access. As similar to other Asian countries, the rising prevalence of westernized lifestyles is likely the most important etiology of increasing CRC incidence in Ho Chi Minh City 6,7 . The westernization of lifestyle, including unhealthy diet, physical inactivity, obesity, alcohol consumption, and smoking, were demonstrated to considerably increase CRC risk $^{19}$. For recent decades, Ho Chi Minh City has been Vietnam's fastest-growing major economic city. This economic transition and urbanization are associated with the westernized lifestyle adoption. The prevalence of overweight and obesity among adolescents in Ho Chi Minh City increased sharply from $5.8 \%$ to $13.7 \%$ during $2002-2004^{20}$. In 2005 , a cross-sectional study on 25-64 years old people in Ho Chi Minh City found that most adults were physically inactive, and only $56.2 \%$ of them achieving the minimum recommendation of "doing 30 minutes moderate-intensity physical activity for at least five days per week" 21 . In Vietnam, the overall alcohol consumption rose strikingly during 2010-2015, and the overall tobacco smoking prevalence has been slowly decreasing but was still high ${ }^{22}$. In addition, another potential etiology is the long-term antibiotic use in early to middle adulthood, which increases the risk colorectal adenoma risk ${ }^{23}$. Vietnam was reported to have a high prevalence of overuse and inappropriate use of antibiotics in hospitals and the community ${ }^{24}$. Parallel with the socioeconomic growth in Ho Chi Minh City, there have been improvements in healthcare accessibility, and more advanced diagnostic techniques introduced (colonoscopy, computed tomography, magnetic resonance imaging, ultrasound, fecal occult blood test, and fecal immunochemical test), which enhanced the detecting rate of CRC.

In accordance with previous data from most parts of the world, our study found that CRC rates were higher in men than in women ${ }^{1}$. The APC analysis revealed that men and women had the CRC rates elevate along with aging, in which the risk was much higher in men than women from the age of 60-64 years old. This discrepancy might result from the higher prevalence of risk behaviors (smoking and alcohol uptake) in men and the protective effect of endogenous estrogens against CRC tumorigenesis in postmenopausal women 25 .

In contrast to many other Asian nations, Ho Chi Minh City experienced a marked increasing CRC incidence trend in the old population than in the younger counterparts. A modest but significant escalating of early-onset CRC was reported in Taiwan, Korea, Japan, and Hong Kong from 1995 to $2014^{26}$. This difference could result from the screening programs mature and improvement in other Asian 
countries while the organised CRC screening has not been implemented in Vietnam until now. Several Asian countries implemented the population-based CRC screening program in recent decades, such as Japan (since 1992), Korea (since 2004), China (since the 2000s), Taiwan (since 2004), Hong Kong (since 2017), Thailand (since 2011), and Singapore (since 2011) ${ }^{27-29}$. The starting age screening age for average-risk individuals in those programs ranged from 40 to 50 years old. Since all CRC cases in Ho Chi Minh City were opportunistic diagnosed, Ho Chi Minh City's CRC incidence might undervalue the true rate of early-onset diagnoses. Together with the overall increase of CRC incidence, particularly in people born from the 1976-1980 period, Ho Chi Minh City might be in need of launching a population-based CRC screening program to early diagnose the $\mathrm{CRC}$ and improve the overall disease outcome.

Regarding anatomical subsite, our study found that rectal cancer had the highest rate rising while the proximal and distal colon increased relatively in both genders. Globally, the CRC subsites trend varied widely. There has been a proximal shift in the CRC subsite distribution in various countries globally, such as the USA (1970-2000), Canada (1964-2004), Japan (1974-1994), Norway (1962-2006), and Luxembourg (1988-1998) 12,30-33. Conversely, this trend has not been observed in several other countries, such as Italy (1984-1998), Iran (1994-2009), and Korea (1999-2009), as the rate increased in all CRC subsites or right-sided cancer did not increase statistically significant ${ }^{34-36}$. The reasons for these conflicting findings remain unclear but might be explained by the complex attributions of risk factors on different tumor segments. A study from Japan demonstrated that heavy tobacco smoking was linked with increased rectal cancer risk in men but not women ${ }^{37}$. This study also indicated that high alcohol consumption was substantially associated with rectal cancer risk in women while increased the risk of colon cancer in either gender insignificantly. Another study on Japanese men reported that alcohol intake was associated with a statistically significant increased risk of distal and rectal cancer, but not proximal cancer $^{38}$. Regarding body mass index, overweight and obesity were reported to elevate colon cancer risk in men but not women ${ }^{37,39}$. Meanwhile, a Korean study reported that a high body mass index was associated with increased risk for proximal colon cancer among women, and a study on Asian women outlined that obesity is positively associated with colon cancer but not rectal cancer ${ }^{40,41}$. Physical inactivity had shown a strong association with the risk of proximal colon cancer in women but not with rectal cancer in both genders ${ }^{42}$. We need further studies to ascertain whether there is a true proximal shift trend of CRC in Vietnam and find associated factors. This is essential in choosing the preferred screening test modality between sigmoidoscopy and total colonoscopy.

The Ho Chi Minh Cancer Registry collected CRC archives from all hospitals in Ho Chi Minh City for a longterm. Its coverage could be representative of CRC epidemiology on a regional scale. However, the present study had some limitations. Associated factor data were not available or lack of completeness, such as family history, heredity cancer syndromes, socio-economic characteristics, the basis of diagnosis test frequency, and cancer staging. The high proportion of the other CRC subsites (26.8\%) might affect the topography analysis accuracy. Many other Asian nations also reported high proportions of overlapping and unspecified lesions of $\mathrm{CRC}^{43}$. Additionally, due to the confounding and mixture among 
age, period, and cohort effects, the interpretations of results should be used with cautions of misleading, particularly at the individual level.

\section{Conclusions}

The CRC incidence in Ho Chi Minh City increased with the more prominent rising rates among men, older people, and rectal subsites. The upward trends of CRC incidence in Ho Chi Minh City could be explained by the increase in westernized lifestyles, long-term antibiotic use, improved diagnostic techniques, and healthcare access. This study's result implied the need of launching a population-based CRC screening program in Ho Chi Minh City. Further studies are needed to determine the CRC risk factors and identify the people at risk for whom a screening program might provide the best benefit.

\section{Abbreviations}

APC: Age-Period-Cohort; ARC: Annual Rate of Change; ASR: Age-Standardized Incidence Rate; Cl: Confidence Interval; CRC: Colorectal Cancer; GSO: General Statistics Office; ICD-O: International Classification of Diseases for Oncology; ICD: International Classification of Diseases; RR: Rate Ratio; SD: Standard Deviation.

\section{Declarations}

\section{Acknowledgements}

We gratefully acknowledge the assistance of the Ho Chi Minh Oncology Hospital, Vietnam.

\section{Author contributions}

DXP, TST, LDM, LTHP and TVN conceived and designed the study. DXP, HDN, AHTP and TVN performed the study and data collections. DXP, HDN, AHTP, TDB, LTHP and TVN analyzed and interpreted the results of data. DXP, HDN, AHTP and TDB drafted the manuscript. TST, LDM, LTHP and TVN revised the manuscript. All authors read and approved the final manuscript.

\section{Competing interests}

The authors declare that they have no competing interests.

\section{Funding}

None

\section{Availability of data materials}

Data from all registries was collected and assessed based on guidelines from the International Agency for Research on Cancer and the International Association of Cancer Registries, adapted to a low and 
middle-income context. Registry data are stored in a computerized data at the Oncology Hospital of Ho Chi Minh City. The dataset includes the following variables: age, sex, diagnosis, year of diagnosis, cancer stage, treatment, and survival status. All authors had no special access privileges in accessing these datasets, which other interested researchers would not have. Age-and-gender population statistics were obtained from census data managed by the General Statistics Office (GSO) of Ho Chi Minh City. Age-andgender population statistics in 1999 for Vietnam were obtained from the Bureau of Statistics of Vietnam. There is no URL for the population data. However, requests for data access can be made to the General Statistics Office of Ho Chi Minh City, contact through email tdnlinh@ump.edu.vn.

\section{Ethics approval and consent to participate}

The study was approved by the Ethics Committee of the Oncology Hospital of Ho Chi Minh City, Vietnam.

\section{Consent for publication}

Not applicable

\section{References}

1. Globocan- The Global Cancer Observatory. Colorectal cancer Source: Globocan 2018.

2. Torre, L. A., Bray, F., Siegel, R. L., Ferlay, J., Lortet-Tieulent, J., \& \& Jemal, A. Global cancer statistics,2012. CA: A. Cancer J. Clin. (2015).

3. Sung, J. J. Y. et al. Increasing incidence of colorectal cancer in Asia: Implications for screening. Lancet Oncology (2005) doi:10.1016/S1470-2045(05)70422-8.

4. Sung, J. Colorectal cancer screening: Its time for action in Asia. Cancer Detection and Prevention (2007) doi:10.1016/j.cdp.2007.01.002.

5. Onyoh, E. F. et al. The Rise of Colorectal Cancer in Asia: Epidemiology, Screening, and Management. Current Gastroenterology Reports (2019) doi:10.1007/s11894-019-0703-8.

6. Center, M. M., Jemal, A., Smith, R. A. \& Ward, E. Worldwide Variations in Colorectal Cancer. CA. Cancer J. Clin. (2009) doi:10.3322/caac.20038.

7. Kuipers, E. J. et al. Colorectal cancer. Nat. Rev. Dis. Prim. (2015) doi:10.1038/nrdp.2015.65.

8. Keum, N. N. \& Giovannucci, E. Global burden of colorectal cancer: emerging trends, risk factors and prevention strategies. Nature Reviews Gastroenterology and Hepatology (2019) doi:10.1038/s41575019-0189-8.

9. Siegel, R. L. et al. Colorectal Cancer Incidence Patterns in the United States, 1974-2013. Journal of the National Cancer Institute (2017) doi:10.1093/jnci/djw322.

10. Feletto, E. et al. Trends in colon and rectal cancer incidence in Australia from 1982 to 2014: Analysis of data on over 375,000 cases. Cancer Epidemiol. Biomarkers Prev. (2019) doi:10.1158/10559965.EPI-18-0523. 
11. Brenner, D. R. et al. Increasing colorectal cancer incidence trends among younger adults in Canada. Prev. Med. (Baltim). (2017) doi:10.1016/j.ypmed.2017.10.007.

12. Larsen, I. K. \& Bray, F. Trends in colorectal cancer incidence in Norway 1962-2006: An interpretation of the temporal patterns by anatomic subsite. Int. J. Cancer(2010) doi:10.1002/ijc.24839.

13. Araghi, M. et al. Changes in colorectal cancer incidence in seven high-income countries: a populationbased study. Lancet Gastroenterol. Hepatol. (2019) doi:10.1016/S2468-1253(19)30147-5.

14. Countries, I., Agency, I. \& America, S. Global Initiative for Cancer Registry Development in Low - and Middle - Income Countries: quality information to support effective cancer control. 4-5 (2011).

15. Fritz A, Percy C, Jack A, Shanmugaratnam K, Sobin L, Parkin DM, W. S. (eds). International classification of diseases for oncology (ICD-O) - 3rd edition, 1st revision. World Heal. Organ. (2000).

16. UNITED STATES, National Cancer Institute \& Division of Cancer Control and Population Sciences. Joinpoint Regression Program. USA: Surveillance Research Program (2020).

17. Kim, H. J., Fay, M. P., Feuer, E. J. \& Midthune, D. N. Permutation tests for joinpoint regression with applications to cancer rates. Stat. Med. (2000) doi:10.1002/(SICI)1097-

0258(20000215)19:3<335::AID-SIM336>3.0.C0;2-Z.

18. Rosenberg, P. S., Check, D. P. \& Anderson, W. F. A web tool for age-period-cohort analysis of cancer incidence and mortality rates. Cancer Epidemiol. Biomarkers Prev. (2014) doi:10.1158/10559965.EPI-14-0300.

19. World Cancer Research Fund / American Institute for Cancer Research. Continuous Update Project Report. Food, Nutrition, Physical Activity, and the Prevention of Colorectal Cancer. Wcrf/Aicr (2011).

20. Hong, T. K. et al. Overweight and obesity are rapidly emerging among adolescents in Ho Chi Minh City, Vietnam, 2002-2004. Int. J. Pediatr. Obes. (2007) doi:10.1080/17477160701520165.

21. Trinh, O. T. H., Nguyen, N. D., Dibley, M. J., Phongsavan, P. \& Bauman, A. E. The prevalence and correlates of physical inactivity among adults in Ho Chi Minh City. BMC Public Health (2008) doi:10.1186/1471-2458-8-204.

22. Nguyen, T. P. et al. Attributable causes of cancer in Vietnam. J. Glob. Oncol. (2020) doi:10.1200/JG0.19.00239.

23. Cao, Y. et al. Long-term use of antibiotics and risk of colorectal adenoma. Gut (2018) doi:10.1136/gutjnl-2016-313413.

24. Thu, T. A. et al. Antibiotic use in Vietnamese hospitals: A multicenter point-prevalence study. Am. J. Infect. Control (2012) doi:10.1016/j.ajic.2011.10.020.

25. Murphy, N. et al. A Prospective Evaluation of Endogenous Sex Hormone Levels and Colorectal Cancer Risk in Postmenopausal Women. J. Natl. Cancer Inst. (2015) doi:10.1093/jnci/djv210.

26. Sung, J. J. Y. et al. Increasing Trend in Young-Onset Colorectal Cancer in Asia: More Cancers in Men and More Rectal Cancers. Am. J. Gastroenterol. (2019) doi:10.14309/ajg.0000000000000133.

27. Navarro, M., Nicolas, A., Ferrandez, A. \& Lanas, A. Colorectal cancer population screening programs worldwide in 2016: An update. World Journal of Gastroenterology (2017) 
doi:10.3748/wjg.v23.i20.3632.

28. Schreuders, E. H. et al. Colorectal cancer screening: A global overview of existing programmes. Gut (2015) doi:10.1136/gutjnl-2014-309086.

29. Sano, Y. et al. Colorectal cancer screening of the general population in East Asia. Digestive Endoscopy (2016) doi:10.1111/den.12579.

30. Cucino, C., Buchner, A. M. \& Sonnenberg, A. Continued rightward shift of colorectal cancer. Dis. Colon Rectum (2002) doi:10.1007/s10350-004-6356-0.

31. Takada, H. et al. Changing site distribution of colorectal cancer in Japan. Dis. Colon Rectum (2002) doi:10.1007/s10350-004-6400-0.

32. Singh, H., Demers, A. A., Xue, L., Turner, D. \& Bernstein, C. N. Time trends in colon cancer incidence and distribution and lower gastrointestinal endoscopy utilization in Manitoba. Am. J. Gastroenterol. (2008) doi:10.1111/j.1572-0241.2007.01726.x.

33. Scheiden, R., Pescatore, P., Wagener, Y., Kieffer, N. \& Capesius, C. Colon cancer in Luxembourg: A national population-based data report, 1988-1998. BMC Cancer (2005) doi:10.1186/1471-2407-5-52.

34. de Leon, M. P. et al. Trend of incidence, subsite distribution and staging of colorectal neoplasms in the 15-year experience of a specialised cancer registry. Ann. Oncol. (2004) doi:10.1093/annonc/mdh224.

35. Omranipour, R., Doroudian, R. \& Mahmoodzadeh, H. Anatomical distribution of colorectal carcinoma in Iran: A retrospective 15-yr study to evaluate rightward shift. Asian Pacific J. Cancer Prev. (2012) doi:10.7314/APJCP.2012.13.1.279.

36. Shin, A. et al. Increasing trend of colorectal cancer incidence in Korea, 1999-2009. Cancer Res. Treat. (2012) doi:10.4143/crt.2012.44.4.219.

37. Shimizu, N. et al. Height, weight, and alcohol consumption in relation to the risk of colorectal cancer in Japan: A prospective study. Br. J. Cancer(2003) doi:10.1038/sj.bjc.6600845.

38. Akhter, M. et al. Alcohol consumption is associated with an increased risk of distal colon and rectal cancer in Japanese men: The Miyagi Cohort Study. Eur. J. Cancer(2007) doi:10.1016/j.ejca.2006.09.020.

39. Shin, C. M. et al. Association Among Obesity, Metabolic Health, and the Risk for Colorectal Cancer in the General Population in Korea Using the National Health Insurance Service-National Sample Cohort. Dis. Colon Rectum (2017) doi:10.1097/DCR.0000000000000876.

40. Shin, A. et al. Site-specific risk factors for colorectal cancer in a korean population. PLoS One (2011) doi:10.1371/journal.pone.0023196.

41. Wong, T. S. H., Chay, W. Y., Tan, M. H., Chow, K. Y. \& Lim, W. Y. Reproductive factors, obesity and risk of colorectal cancer in a cohort of Asian women. Cancer Epidemiol. (2019) doi:10.1016/j.canep.2018.10.016.

42. Moradi, T. et al. Occupational physical activity and risk for cancer of the colon and rectum in Sweden among men and women by anatomic subsite. Eur. J. Cancer Prev. (2008) 
doi:10.1097/CEJ.0b013e3282b6fd78.

43. Cancer incidence in five continents. Volume IX. IARC Sci. Publ. (2008) doi:10.1016/00139351(78)90107-x.

\section{Tables}

Table 1 Characteristics of CRC patients in Ho Chi Minh City, 1996-2015. 
1996-2000

(\%)
2001-2005 (\%)

2006-2010 (\%)

2011-2015

(\%)

\section{Gender}

Total

2119

2349

3335

5135

Men

1042 (49.2)

1266 (53.9)

1775

(53.2)

Women

1077 (50.8)

$1083(46.1)$

1560

(46.8)

\section{Age at diagnosis}

Total

$59.5 \pm 15.6$

$60.6 \pm 15.8$

$59.5 \pm$ 15.0

Men

$59.3 \pm 15.9$

$59.7 \pm 15.7$

$58.7 \pm$

15.0

Women

$59.7 \pm 15.4$

$61.5 \pm 15.9$

$60.3 \pm$ 14.9

\section{Age group (years) Men}

$<50$

$306(29.4)$

$336(26.5)$

471

(26.5)

50-64

277 (26.6)

368 (29.1)

640

(36.1)

65-79

378 (36.3)

467 (36.9)

527

(29.7)

$80+$

81 (7.8)

$95(7.5)$

$137(7.7) \quad 234(8.1)$

\section{Age group (years)}

Women

$<50$

$294(27.3)$

$247(22.8)$

337

(21.6)

$50-64$

301 (27.9)

$320(29.5)$

578

(37.1)

65-79

$398(37.0)$

$393(36.3)$

505

(32.4)

$80+$

$84(7.8)$

$123(11.4)$

$140(9.0) \quad 226(10.0)$

\section{Topography}

Distal

$390(18.4)$

409 (17.4)

701

(21.0)

Proximal

$488(23.0)$

475 (20.2)

689

(20.7)

2885 (56.2)

2250 (43.8)

$60.3 \pm 14.3$

$59.9 \pm 14.1$

$60.9 \pm 14.5$

609 (21.1)

1178 (40.8)

864 (29.9)

$456(20.3)$

$872(38.8)$

$696(30.9)$

803 (15.6)

809 (15.8) 


\begin{tabular}{|c|c|c|c|c|}
\hline Rectum & $782(36.9)$ & 814 (34.7) & $\begin{array}{l}1243 \\
(37.3)\end{array}$ & $1873(36.5)$ \\
\hline Others & $459(21.7)$ & $651(27.7)$ & $\begin{array}{l}702 \\
(21.0)\end{array}$ & $1650(32.1)$ \\
\hline \multicolumn{5}{|l|}{ Morphology } \\
\hline Adenocarcinoma & 1345 (94.7) & $1407(95.5)$ & $\begin{array}{l}2158 \\
(96.1)\end{array}$ & $3299(98.2)$ \\
\hline $\begin{array}{l}\text { Adenosquamous } \\
\text { carcinoma }\end{array}$ & $0(0.0)$ & $0(0.0)$ & $0(0.0)$ & $1(0.0)$ \\
\hline $\begin{array}{l}\text { Mucinous } \\
\text { adenocarcinoma }\end{array}$ & $41(2.9)$ & $47(3.2)$ & $57(2.5)$ & $42(1.3)$ \\
\hline $\begin{array}{l}\text { Signet-ring cell } \\
\text { carcinoma }\end{array}$ & $21(1.5)$ & $15(1.0)$ & $19(0.8)$ & $9(0.3)$ \\
\hline Small cell carcinoma & $0(0.0)$ & $0(0.0)$ & $1(0.0)$ & $0(0.0)$ \\
\hline $\begin{array}{l}\text { Squamous cell } \\
\text { carcinoma }\end{array}$ & $13(0.9)$ & $4(0.3)$ & $11(0.5)$ & $7(0.2)$ \\
\hline $\begin{array}{l}\text { Undifferentiated } \\
\text { carcinoma }\end{array}$ & $0(0.0)$ & $0(0.0)$ & $0(0.0)$ & $0(0.0)$ \\
\hline
\end{tabular}

Table 2 Time trends of CRC incidence in Ho Chi Minh City, 1996-2015. 


\begin{tabular}{|lllll|}
\hline \multicolumn{1}{|l}{$1996-2000$} & $2001-2005$ & $2006-2010$ & $2011-2015$ \\
\hline Overall & & & & \\
\hline Crude rate & $8.1(7.7-8.4)$ & $7.4(7.1-7.7)$ & $9.2(8.9-9.5)$ & $13.7(13.3-14.0)$ \\
\hline ASR* & $10.5(10.1-11.0)$ & $9.8(9.4-10.3)$ & $11.8(11.4-12.2)$ & $17.9(17.4-18.5)$ \\
\hline ARC & $1.5(-9.1-13.2)$ & $1.2(-19.4-27.0)$ & $11.8(1.3-23.3)$ & $8.0(0.2-16.3)$ \\
\hline Men & & & & \\
\hline Crude rate & $8.2(7.8-8.8)$ & $8.3(7.9-8.8)$ & $10.2(9.8-10.7)$ & $16.0(15.4-16.6)$ \\
\hline ASR* & $12.6(11.8-13.4)$ & $12.8(12.1-13.6)$ & $15.0(14.2-15.7)$ & $24.6(23.6-25.6)$ \\
\hline ARC & $1.5(-14.8-20.9)$ & $0.0(-25.3-33.9)$ & $10.2(-2.6-24.7)$ & $10.4(1.5-20.1)$ \\
\hline Women & & & & $11.5(11.0-12.0)$ \\
\hline Crude rate & $7.9(7.4-8.4)$ & $6.6(6.2-7.0)$ & $8.3(7.9-8.7)$ & $13.4(12.8-14.0)$ \\
\hline ASR* & $9.2(8.7-9.8)$ & $7.8(7.3-8.3)$ & $9.6(9.1-10.1)$ & $6.2(-2.1-15.1)$ \\
\hline ARC & $1.7(-6.4-10.6)$ & $1.3(-17.4-24.2)$ & $13.6(3.6-24.6)$ & \\
\hline
\end{tabular}

*Standardization to Segi's world population, per 100,000 population

Numbers in brackets represent the $95 \% \mathrm{Cl}$

Figures 

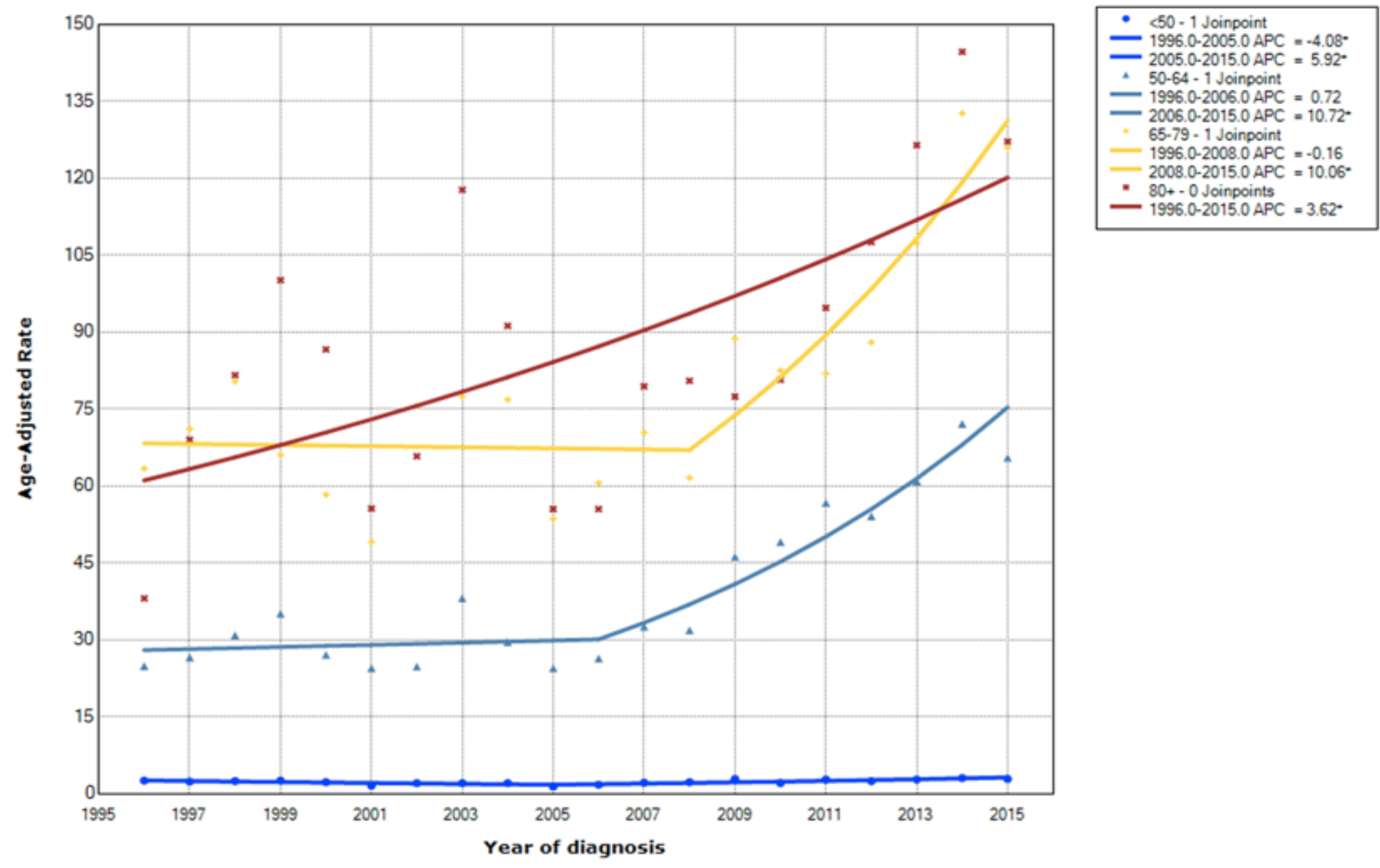

Figure 1

Trends in the ASR (per 100,000) of CRC in Ho Chi Minh City, 1996-2015 by age groups.
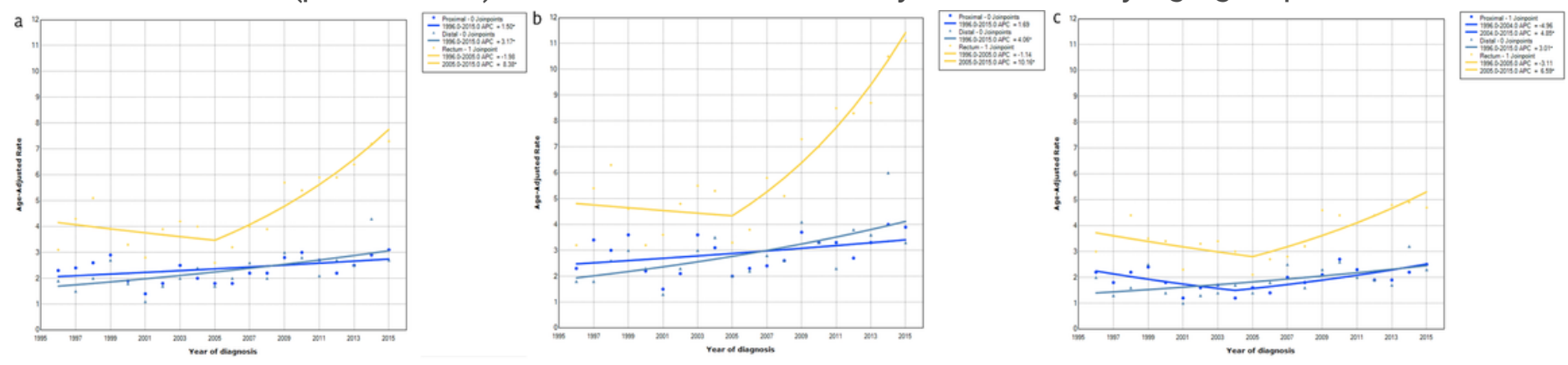

Figure 2

Trends in the ASR (per 100,000) of CRC by anatomical subsites in Ho Chi Minh City, 1996-2015. (a): Overall, (b): Men, (c): Women 

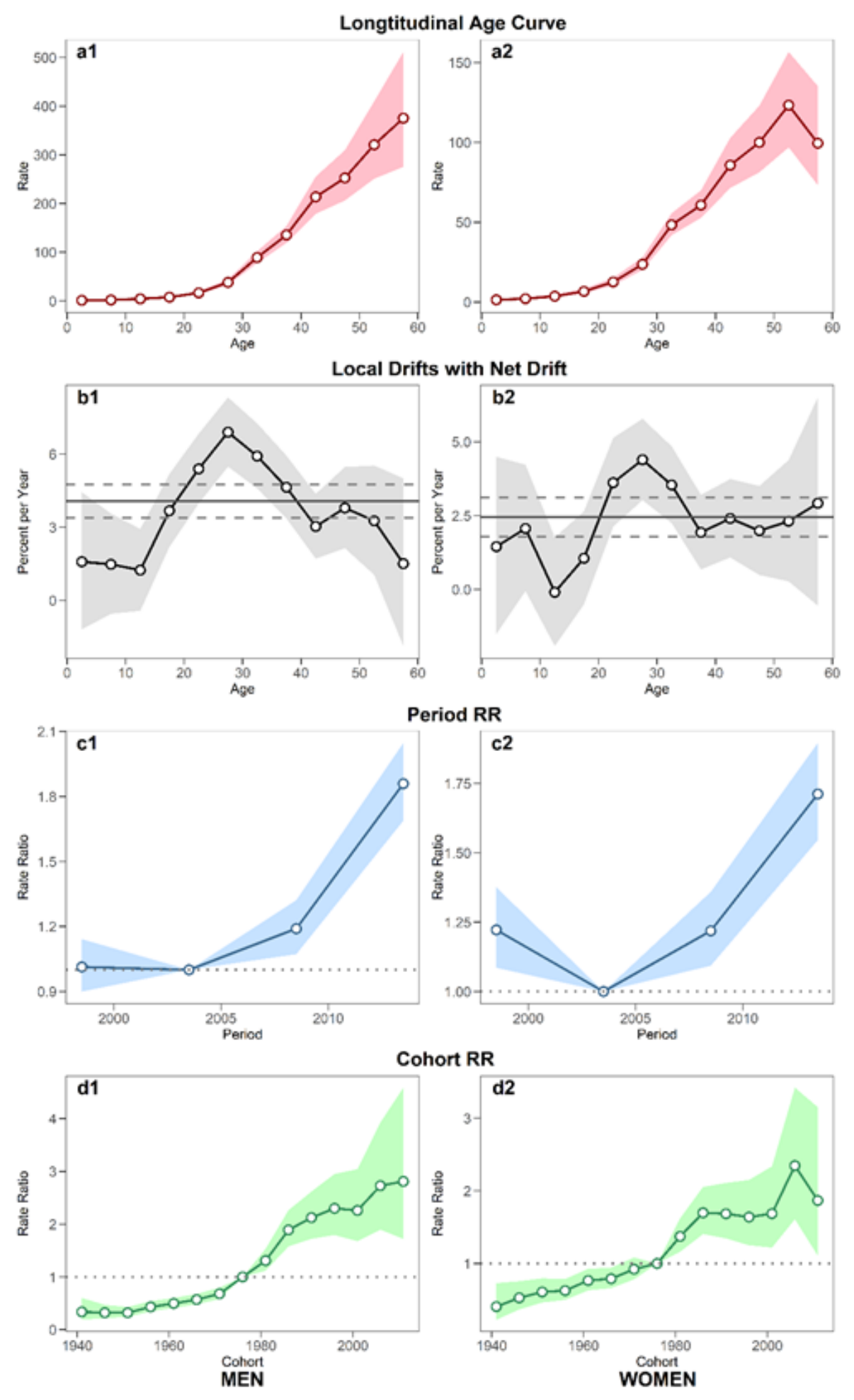

\section{Figure 3}

APC analysis of CRC incidence in men and women in Ho Chi Minh City, Vietnam during 1996-2015. (a1), (a2): Longitudinal age curve and corresponding 95\% confidence intervals (pink area) (b1), (b2): Local drifts with net drift and corresponding 95\% confidence intervals (grey area) (c1), (c2): Period RR and corresponding 95\% confidence intervals (blue area) (d1), (d2): Cohort RR and corresponding 95\% confidence intervals (green area) 


\section{Supplementary Files}

This is a list of supplementary files associated with this preprint. Click to download.

- SupplementaryTable.docx 